\title{
CONTROL OF LOW-RESOLUTION SCANNING OF OVARIAN TUMOR STROMAL COMPARTMENT
}

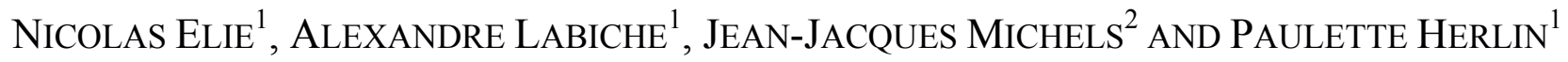 \\ ${ }^{1}$ Groupe Régional d'Etudes sur le Cancer, Equipe d'Accueil Universitaire 1772, Centre de Lutte Contre le \\ Cancer François Baclesse, F. 14076 Caen, France; ${ }^{2}$ Service d'Anatomie Pathologique, Centre de Lutte Contre \\ le Cancer François Baclesse, F. 14076 Caen, France. \\ e-mail: n.elie@tiscali.fr, a.labiche@baclesse.fr, michels@baclesse.fr, p.herlin@baclesse.fr \\ (Accepted March 15, 2005)
}

\begin{abstract}
The active search for markers of prognostic impact and therapeutic response is of crucial importance in oncology. The amount of stroma is a potential indicator with a significant impact on tumor growth. To solve the problems linked to tumor heterogeneity and, hence, of quantization at the microscopic level, we developed an image-analysis strategy dedicated to estimating the proportion of tumor connective tissue in whole histological sections at low resolution. 2,700-dot-per-inch numerical images were acquired with a calibrated slide scanner and automatically analyzed. We performed a quality control in order to evaluate the efficiency of the proposed method of analysis at the selected resolution. The point-counting method and the interactive drawing of stroma limits on equivalent mosaic microscopic images were "the gold standards." The proportions of stroma estimated on microscopic images (magnification with a $4 \times$ objective corresponding to 16,200 dots per inch) and on slide scanner images (2,700 dots per inch) through point counting stereology or manual delineation were equivalent $(\mathrm{p}<0.0001)$. The results obtained through automatic image processing of scanner images were also close to the gold standard ( $95 \%$ correlation, $p<0.0001)$. The proposed method makes it possible to estimate the stroma amount of ovarian carcinomas in whole histological sections with a simple, fast, and low-cost acquisition device.
\end{abstract}

Keywords: automatic image analysis, ovarian cancer, quality control, stroma, slide scanner.

\section{INTRODUCTION}

Several recent studies have emphasized that the functional association between tumor cells and their macro- and micro-environments plays an important role in the tumoral process (Bhowmick et al., 2004; Mueller and Fusenig, 2004). It is now well established that a tumor can be considered like an organ, with permanent interactions between tumor cells, stroma cells, and the extracellular matrix, evolving during the progression of the disease (Bissell and Radisky, 2001; Liotta and Kohn, 2001; Braga, 2002; Yamada and Kemler, 2002). A better knowledge of these interactions is fundamental for the development of new therapeutic protocols (Matrisian et al., 2001) and strategies aimed at overcoming tumor cell resistance (Zhu et al., 1999). In this context, many efforts are being made in order to appraise the mechanism of tumor neovascularization and to develop an effective therapy against angiogenesis (Sokoloff and Chung, 1998; Weiss, 2000; Hicklin et al., 2001; Kerbel and Folkman, 2002). However, the tumor stroma is not composed solely of blood vessels. It is a much more complex tissue, often compared to the granulation tissue developed during wound healing (Bissell and Radisky, 2001) and composed of several cellular types (including fibroblasts and myofibroblasts) involved in the synthesis of the intercellular matrix, a reserve of growth factor, and degradation enzymes (Song et al., 2000; Seiki, 2002). Inside the stroma a transit of inflammatory cells also takes place, enabling tumor cell elimination as well as control of various events, such as degradation of the extracellular matrix, vascularization, and tumor progression (Kamate et al., 2002; Shacter and Weitzman, 2002).

The stroma of solid tumors can be analyzed at various levels, ranging from the molecular level up to the macroscopic level, in order to study potential prognostic factors. The volume percentage of stroma in several locations, such as ovarian tumors, seems to have a prognostic impact or ability to foretell the response to treatment (Shapiro et al., 1992; Deering et al., 1994; Katsoulis et al., 1995; Brugghe et al., 1998; Anttila et al., 2000). Nevertheless, the complex morphology of cancer and tissue heterogeneity constitute a major problem when one wishes to quantify markers in 
histological sections (Konemann et al., 2000). Estimations of stromal compartment are often obtained in a manual or semiautomatic way, in restricted microscopic fields, visually selected and considered representative of the whole tumor section (Schipper et al., 1991; Shapiro et al., 1992; Deering et al., 1994; Katsoulis et al., 1995; Brugghe et al., 1998). This approach presents several drawbacks: slowness of the analysis, inter- and intra-observer variability, and poor statistical significance because the microscope makes it possible to view only a small part of the section. The subjective choice of the areas analyzed may provide spurious results if the sampling is not performed correctly. It is often necessary to measure numerous fields in order to obtain a reliable and reproducible estimate, but the number of fields that should be observed is highly dependant on the degree of heterogeneity. Processing an image of the whole histological section would provide an ideal solution. However, the high cost and complexity of the devices needed limits their introduction and distribution to routine pathology departments.

The actual performances of numerical lowresolution acquisition procedures, image-processing strategies, and computer hardware allow an "in situ" analysis of the whole histological section (Kim et al., 2003; Krout et al., 2002; Chantrain et al., 2003). In this context, the present article describes a simple, inexpensive, and fast image-acquisition and automatic image-processing method aimed at evaluating the stromal compartment of ovarian tumors. The efficiency of the proposed method is also established on the basis of quality control of the chosen resolution (scanner versus microscope) and method of analysis (automatic procedure versus interactive estimation).

\section{MATERIALS AND METHODS}

\section{CANCER SPECIMENS AND TISSUE PREPARATION}

Tissue samples were obtained from 20 surgical specimens of human ovarian adenocarcinomas (serous and mucinous types). Samples were fixed in buffered formalin and embedded in paraffin. Then $5-\mu \mathrm{m}$-thick histological sections were fixed on polylysine-coated slides for histochemical staining.

\section{STAINING}

Histological sections were deparaffinized and rehydrated, and then nuclei were stained with Mayer hematoxylin (Biogenex, Menarini Diagnostics, Antony, France) for $5 \mathrm{~min}$. After washing in $\mathrm{pH}=7$ water for $15 \mathrm{~min}$, the sections were dehydrated and the collagen was stained for $30 \mathrm{~min}$ with saffron (Microm, Francheville, France). Finally, the stained sections were mounted in an Entellan mounting medium.

\section{IMAGE ACQUISITION}

A single numerical image of the whole histological section of each specimen was recorded, using a Nikon Super Coolscan 8000 ED slide scanner (Nikon, Champigny sur Marne, France), provided with a special medical slide holder (FH-8G1, Nikon, Champigny sur Marne, France). The resolution used was 2,700 dots per inch, 1 pixel covering an area of $88 \mu \mathrm{m}^{2}$. Using this device and these specifications, it is possible to scan a maximum tissue section area of $11 \mathrm{~cm}^{2}$, which corresponds to a numerical image of $4,890 \times 2,550$ pixels and requires 37 megabytes of hard disc space. In the present study, tumor sections had a mean area of about $2 \mathrm{~cm}^{2}$, corresponding to 20 megabytes. The approximate scanning time needed per image was $3 \mathrm{~min}$, with an AMD Athlon XP1700 personal computer, provided with 256 megabytes of random access memory. The true-color images (24 bits RGB color) were saved in uncompressed tagged image file format (TIFF).

In order to compare stroma detection at scanner and microscopic levels, a small portion of the scanner image was selected and a microscopic mosaic image of strictly the same region was built.

For this purpose, an automatic procedure was developed in our laboratory to drive a fully automatic microscope (Olympus AX70, Olympus, France) coupled to a 3CCD video camera (Color Vision Camera Module, Sony, France) and a frame grabber (Matrox Meteor, $768 \times 576$ pixels). The software, developed in Visual $\mathrm{C}++$ (Microsoft Corporation, Redmont, WA, USA), makes it possible to draw a region of interest (ROI) onto the scanner image, to choose a microscope magnification, and to start acquisition of neighboring fields. The focus is set automatically and a microscopic mosaic image is then built (Thuillier et al., 2001).

In this study, 20 microscopic mosaic images were obtained, corresponding to the relevant ROIs selected on the scanner image for quality control. These images were composed of an average of 12 microscopic fields, corresponding to an area of at least $16 \mathrm{~mm}^{2}(4 \mathrm{~mm} \times$ $4 \mathrm{~mm}$, objective $4 \times$, resolution of $16,256 \mathrm{dpi}, 1$ pixel covering $2.44 \mu \mathrm{m}^{2}$ ). 


\section{STANDARDIZATION OF IMAGE ACQUISITION}

Using a special holder, the Nikon slide scanner enables the user to directly scan histological glass slides. However, if no adjustment were pre-established, the intensity of the images obtained would be too high and the colors would be of poor quality. The scanner was calibrated with reference to the "Wide Gamut RGB" International Color Consortium (ICC) color-space profile for positive films (Adobe Systems Incorporated, San Jose, CA, USA) before applying a special setting for histological sections stained with Mayer hematoxylin and saffron, using Nikon Scan 3.1 software (Nikon, Champigny sur Marne, France). Using the "Curves" tools, pixels of gray images (red, green, and blue components associated) exhibiting an intensity of 128 to 240 gray levels were redistributed between 0 and 255. Using the "Color Balance" tool, it was possible to darken the slide scanner image. A new relationship was obtained with a value of -40 for brightness (scale between -100 and +100 ). To remove the nuance of pink present on the image, we decreased the values of the red component by 20 levels of gray. The acquisition profile was recorded and used for all acquisitions.

\section{AUTOMATIC ANALYSIS OF TISSUE SECTIONS}

Images of histological sections were analyzed using an image-processing toolbox (Aphelion, ADCIS S.A, Hérouville Saint-Clair, France). Operators were chained as a macro-application, allowing a rapid, reproducible, and easy-to-use image-processing routine. The approximate processing time needed was one minute for eight images.

An optional interactive procedure was added in order to select tumor tissue and to discard normal remaining tissue, mucus, and necrosis from the whole histological section when needed. This optional step may be used also to eliminate folds and artifactual large color deposits.

Tissue detection. Adaptive thresholding (momentpreserving thresholding according to Tsai (1985)) was used to detect the whole tissue section on the blue component of the color image, which exhibits the best contrast between tissue and background.

Collagen detection. In order to obtain yellow pixels, the Hue-Saturation-Intensity color mode was used (Novak and Shafer, 1992). Hue image is encoded between zero and six for the six primary colors, yellow ranging at one. Therefore, a fixed threshold was settled on the hue image (between 0 and 1) in order to obtain a binary image of the yellow pixels.

Stromal fraction computation. Logical "AND" operators were then applied between the mask image of the tumor and binary images of tissue and stroma in order to finally compute the stroma-to-tumor ratio.

\section{QUALITY CONTROL PROCEDURE}

Two quality controls were performed: a quality control of the interactive stromal-fraction estimation at low resolution provided by the scanner (comparison with the "gold standards": point counting or manual drawing at microscopic level) and a quality control of the automatic image processing (comparison with manual delineation of stroma limits) (Fig. 1).

The work was done on 20 ROIs of tumor sections. Each detail was acquired with both the slide scanner and the automatic microscope. We restricted the quality control to a very small area of $16 \mathrm{~mm}^{2}$ on average. A quality control of the whole section (average of $2 \mathrm{~cm}^{2}$ ) would have meant a ten-fold higher workload in order to create and stitch together the mosaic image and to manually delineate the stroma outlines; furthermore, it would have been difficult to process the resulting 150 megabytes or more of digital images. PaintShop Pro 7 (JASC Software, Minneapolis, MN, USA) was used to draw limits of stroma and tumor tissue, at zoom $1 \times$ for microscopic mosaic images $(70 \mathrm{~min})$ and at zoom $5 \times$ for scanner images (10 min). Estimation of the stroma proportion was also achieved with a stereological point-counting frame for microscopic mosaic images (Brugghe et al., 1998). A minimum 100-point grid was applied to each image with a $280 \mu \mathrm{m}$ interval between points. The first point was placed randomly. Five stereological stroma proportion estimations were carried out repeatedly for each image $(10 \mathrm{~min}$ per image). The Aphelion toolbox was used (ADCIS S.A, Hérouville Saint-Clair, France) for automatic image analysis and computation.

Pearson's coefficients of correlation between the results obtained at high- or low-resolution were calculated, using Xlstat 6.1.8 (Addinsoft, France). P values $<0.05$ were considered statistically significant. The error bars in Fig. 3 indicate the standard deviation (SD) of the five different stereological estimates of stromal fraction. 


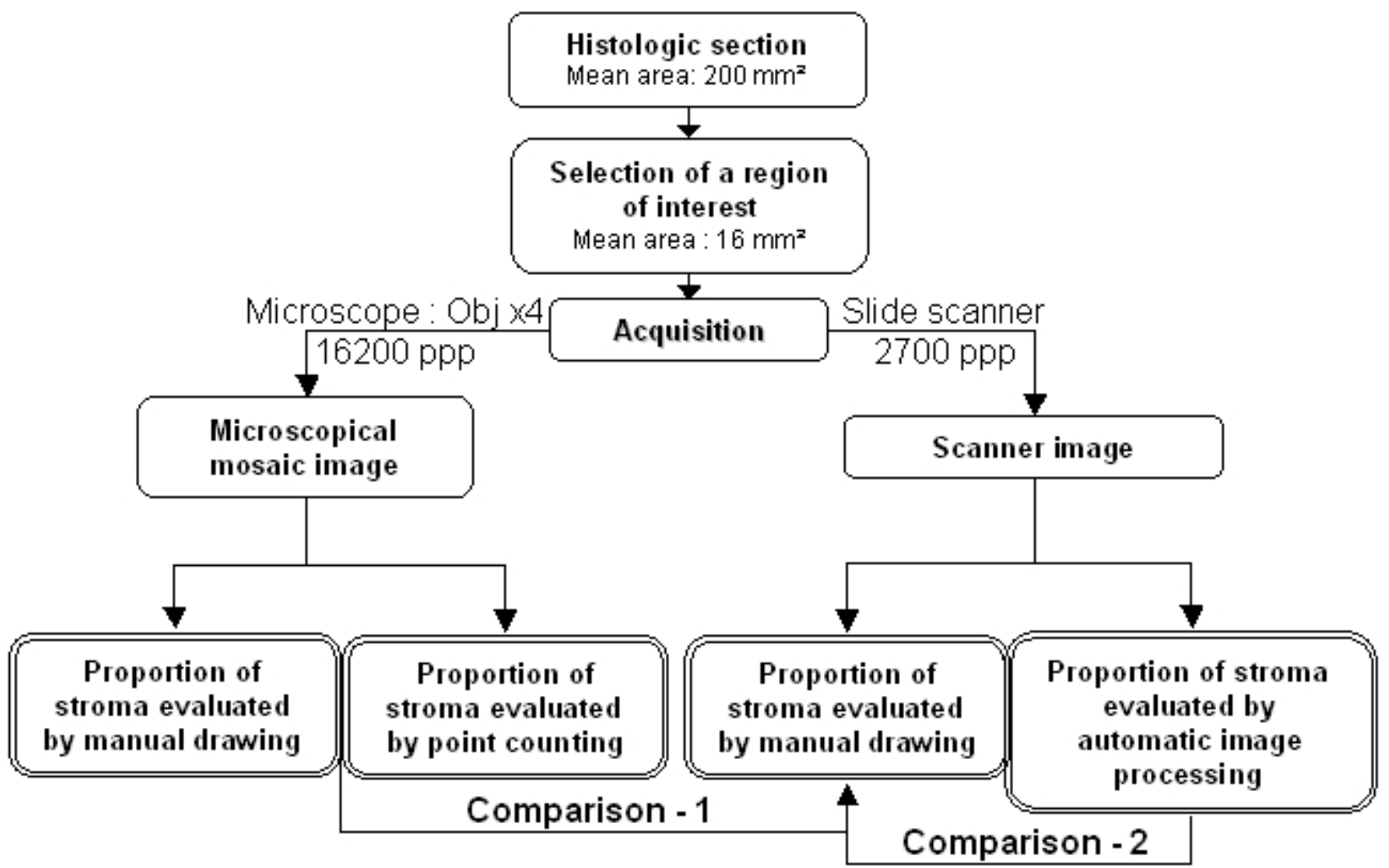

Fig. 1. Presentation of the quality control strategy. The proportions of stroma obtained in scanner images were compared to those yielded after interactive drawing and point counting on microscopic mosaic images covering the same territories (comparison: 1). The values resulting from interactive drawing in the scanner images were then compared to those obtained through automatic image processing (comparison: 2 ).

\section{RESULTS}

The use of a slide scanner provides low-resolution virtual slides of ovarian tumors (i.e., images of the whole histological sections, Fig. 2a) in a single step. The selected acquisition resolution of $2,700 \mathrm{dpi}$ associated with bichromic staining of the tumor tissue enables the user to easily identify the clusters of tumor cells and the connective tissue compartment (Figs. 2b, c, and d) visually or automatically and to estimate the stromal fraction of the tumors.

On whole sections, the scanner makes it possible to gain much acquisition time: $3 \mathrm{~min}$ versus $3 \mathrm{~h}$ for a microscope (Table 1). Furthermore, the lower number of pixels dramatically reduces the storage volume as well as the processing time expense (Table 1).

Table 1. Comparison of time and hard disk space required to obtain an image of $0.16 \mathrm{~cm}^{2}$ and an image of the whole histological section with an automatic microscope and a slide scanner.

\begin{tabular}{lcccc}
\hline & \multicolumn{2}{c}{ For an area of $0.16 \mathrm{~cm}^{2}$} & \multicolumn{2}{c}{ For a whole section of $2.00 \mathrm{~cm}^{2}$} \\
\hline Time & Hard disk space & Time & Hard disk space \\
\hline $\begin{array}{l}\text { Scanner image } \\
\begin{array}{l}\text { Microscopical } \\
\text { mosaic image }\end{array}\end{array}$ & $0.01 \mathrm{hr}$ & 0.5 megabytes & $0.05 \mathrm{~h}$ & 20 megabytes \\
\hline
\end{tabular}

$\S$ With the systems presented in materials and methods; * Numbers obtained by interpolation. 


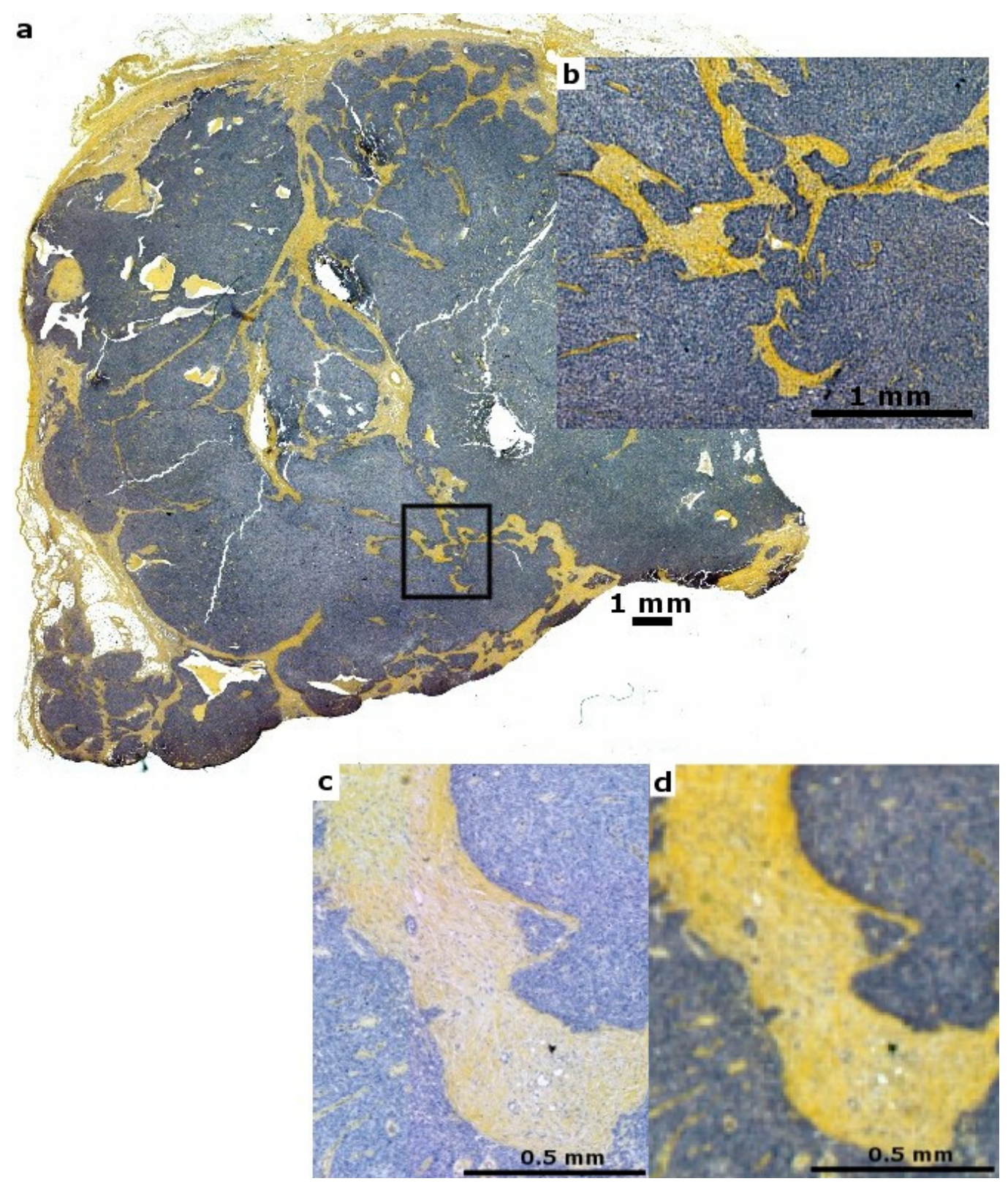

Fig. 2. A 2,700-dpi color image obtained after acquisition with the calibrated slide scanner. (a) This image corresponds to the whole histological section of an ovarian carcinoma, stained with hematoxylin and saffron. (b) Detail of image $2 a$ (black box) at full resolution. Tumor structures can easily be recognized (stroma and cancer cells). (c-d) Comparison between mosaic microscopic image and slide scanner image that preserves color information and limits of structures.

The five stroma-proportion estimates obtained by point counting on each microscopic mosaic image were very close, and the proportions estimated using interactive drawing on the microscopic mosaic images were equivalent to the point-counting method. The results obtained by both methods were taken as reference values.

As a result, the proportions of stroma estimated on the scanner images through interactive drawing strongly correlated with the proportions estimated on microscopic mosaic images, through either interactive drawing or point counting $(88 \%, \mathrm{p}<0.0001 ; 83 \%$, $\mathrm{p}<0.0001$; Fig. 3). Regarding scanner images, 13 cases out of 20 showed proportions of stroma close to the values estimated by stereology and 17 cases out of 20 values comparable with those obtained by drawing on microscopic mosaic images. Only the results of images 11 and 20 were notably different. There was agreement in $90 \%$ of the cases. 
The non-relevance of images 11 and 20 was due to a problem with saffron staining. This stain slightly marked the cytoplasm of cancer cells, as was obviously shown at the microscopic level but was impossible to visually distinguish on the scanner image.

The proportions of stroma estimated on scanner images through interactive drawing and image analysis had a high $(95 \%)$ coefficient of correlation $(p<0.0001$; Fig. 4). Fig. 5 shows the quality of image processing when there was no spurious staining.
The standard deviation computed from the four values obtained for each case through point counting, interactive drawing (microscope and slide scanner), and automatic image analysis was small, because it ranged between $1 \%$ and $4 \%$ for 12 images out of 20 (images 1, 2, 3, 5, 6, 8, 9, 10, 13, 15, 16, 17) and between $5 \%$ and $8 \%$ for 6 images (images $4,7,11,12$, 14, 19). For two images only (images 18 and 20), the standard deviation reached $10 \%$ and $16 \%$, respectively.

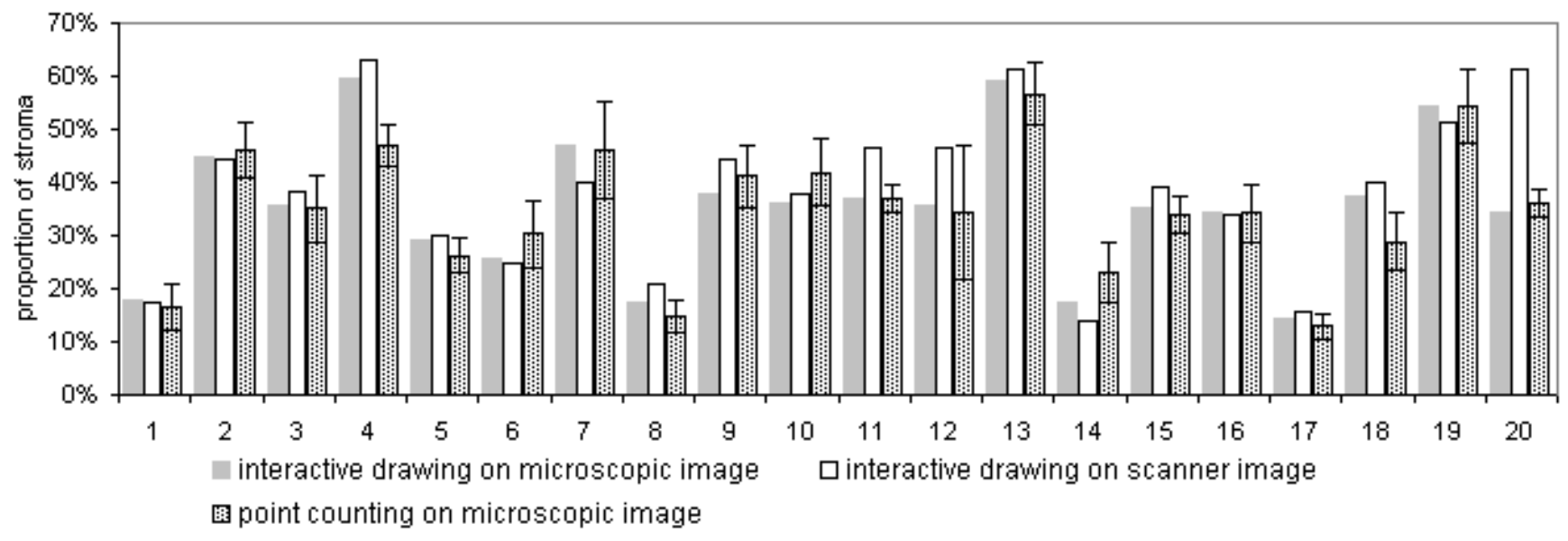

Fig. 3. Comparison of the stroma proportions obtained through interactive drawing on the microscopic image and the equivalent scanner image. The proportions of stroma obtained through point counting are also provided; these correspond to the mean of 5 estimates carried out on the same images (standard deviation in vertical bars).

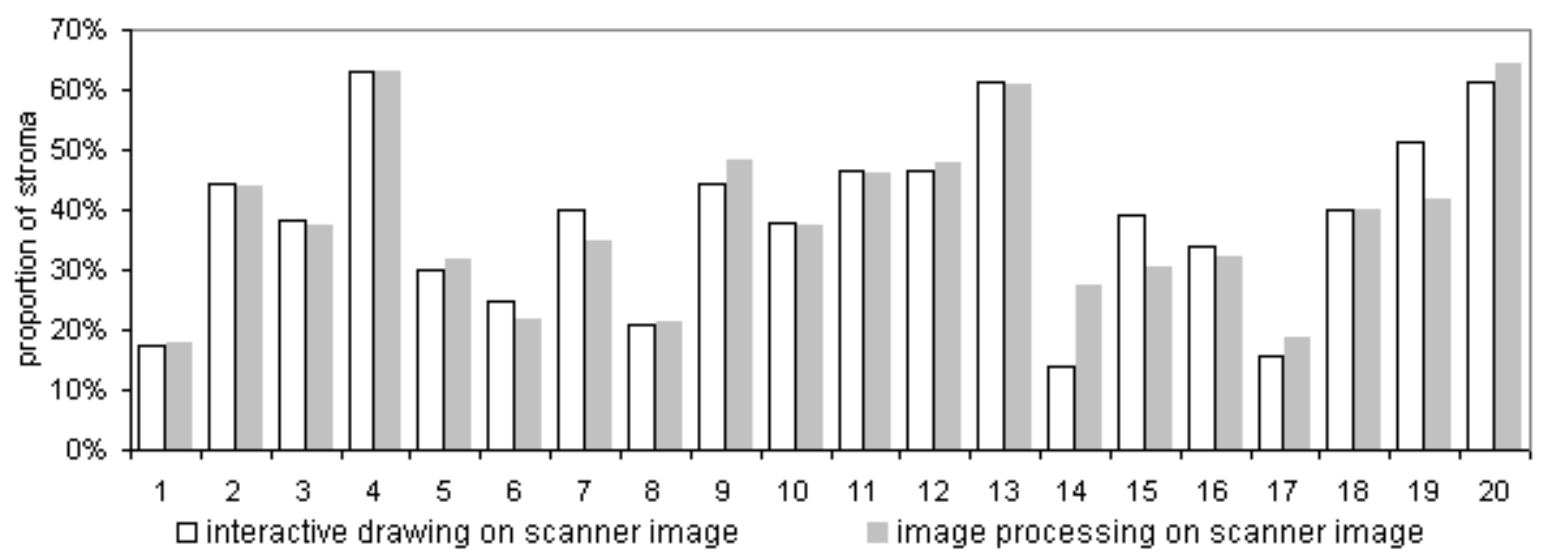

Fig. 4. Comparison between the proportions of stroma obtained on the scanner images through interactive drawing and image processing. 


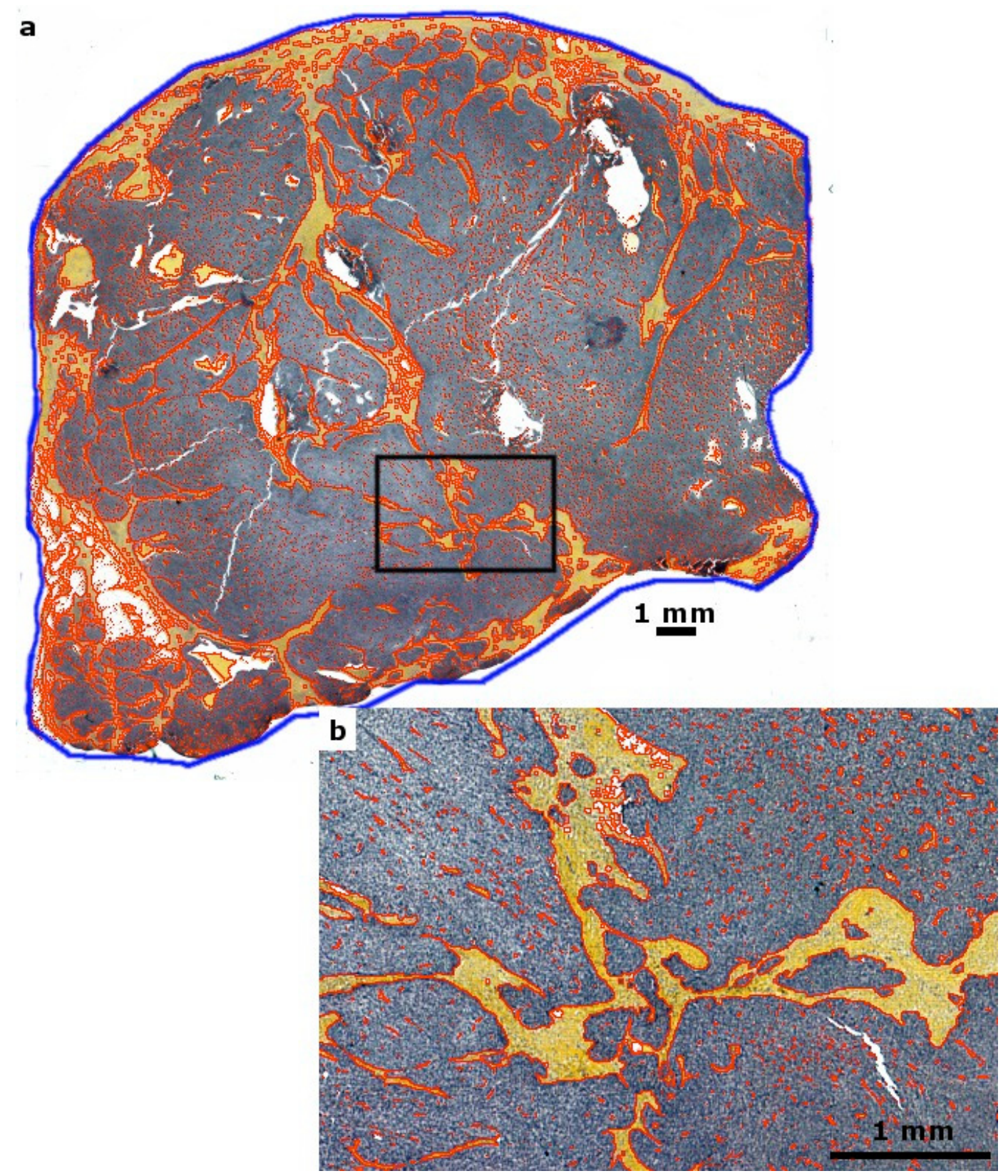

Fig. 5. Limits of automatically detected cancer stroma (in red) and tumor tissue (in blue) are superimposed onto the color image acquired at 2,700 dpi, using the slide scanner. (a) This image corresponds to the whole histological section shown in Fig. 2. (b) Detail of image 5 a (black box) at full resolution.

\section{DISCUSSION}

The goal of the proposed method was to obtain a single image of a whole histological section of a tumor that can be analyzed by automatic image processing. The proportion of stroma in ovarian tumors can be assessed in a simple, fast, and inexpensive way.

Recording of scanner images of histological sections has already been proposed by other authors (Yang et al., 1998; Schmitt and Eggers, 1999; Castro et al., 2000; Krout et al., 2002; Chantrain et al., 2003), but to the best of our knowledge none of these researchers have used it for quantification of stroma proportion.

In order to easily identify the architecture of a tumor, classical staining with hematoxylin, eosin, and saffron must be modified. The staining of cytoplasm with eosin was avoided in order to obtain only three compartments on the image to be analyzed: background in white, nuclei in blue, and stromal collagen in yellow. This simplified bichromic staining procedure is reproducible when staining time and concentrations 
are taken into account. In this study, 2 out of 20 cases presented a non-specific staining of cytoplasm. This observation points out the necessity of checking the staining quality under the microscope before scanner image acquisition and analysis.

Acquisition conditions have been optimized and used for all images to insure restitution of the same colors and equal intensity. The defined settings can be saved, exported, and used with other slide scanners from the same manufacturer. This is easier than using the tedious and hazardous interactive setting for microscopic image acquisition. The slide scanner provides a homogeneous image illumination that guarantees reproducibility of the results obtained by automatic image processing. In addition, the use of a commercially available toolbox for software building makes the reproducibility and exportability of the automatic image-processing protocol possible. In our hands, the method presented here made it possible to obtain an image of a whole histological section in three minutes, versus more than three hours for our automatic microscope, and used storage space twelve times smaller than a $4 \times$ RGB virtual microscopic slide. The system is also ten times cheaper than a microscope equipped with an automatic moving stage and a tri-CCD camera. The resolution obtained with the scanner is six times smaller than the resolution delivered by our microscope acquisition equipment, but obviously sufficient to distinguish the architecture of the tumor. The major drawback of the scanner resolution used $(2,700 \mathrm{dpi})$ is the impossibility of precisely identifying cell categories and individualizing their cytostructures, which may trigger measurement errors in the case of spurious staining. Another problem may be encountered when there is an important inflammatory infiltrate. The corresponding areas may be considered to be clusters of cancer cells.

Concerning the proposed image-processing strategy, the user need only adhere to a fully automatic protocol, apart from delineating tumor tissue limits, when residual normal tissue exists and must be discarded. All the other steps are automatic. In the majority of the published protocols, segmentation by thresholding is obtained through an interactive setting on microscopic mosaic images (Johansson et al., 2001) or scanner images (Chantrain et al., 2003), which cannot guarantee intra- and interobserver reproducibility. In the present study, two fixed thresholds were used. The choice of this segmentation method is allowed because the scanner provides homogeneous and steady illumination conditions for all images, which guarantees good reproducibility of the tumor tissue and stroma limit extractions. Using this simple method, the areas of stroma and tissues detected by automatic image processing are equivalent to areas obtained through interactive drawing or a point-counting estimation on microscopic mosaic images. Nevertheless, it is important to stress that this method gives only an estimate of the stroma proportion, because some areas of connective tissue may be too small to be detected. Indeed, stroma may be considered a fractal structure (Cross, 1997; Smolle, 1998) because for each increasing resolution of a scanner or a microscope, one will detect new regions of connective tissue. The purpose of the method presented here is only to obtain a reliable approximation of the quantity of stroma in a tumor.

The slide scanner can be used also to automatically evaluate immunolabeled vessels (Chantrain et al., 2003; Kim et al., 2003) and nuclei (Elie et al., 2003) through specific automatic image-processing protocols. These simple and inexpensive approaches can potentially help in swiftly and reproducibly identifying biological markers of prognostic significance in whole sections as well as in tissue arrays (Signolle et al., 2003) of large tumor series.

In conclusion, the proposed method allows a simple, fast, and reproducible estimation of the stroma amount in ovarian carcinomas, in whole histological sections. Nevertheless, the staining quality must be checked under the microscope before performing automatic image acquisition and processing.

\section{ACKNOWLEDGMENTS}

Nicolas Elie received a MESR fellowship. The authors would like to thank any person who has contributed to this work and particularly Yves Denoux and Catherine Moricet.

\section{REFERENCES}

Anttila MA, Tammi RH, Tammi MI, Syrjanen KJ, Saarikoski SV, Kosma VM (2000). High levels of stromal hyaluronan predict poor disease outcome in epithelial ovarian cancer. Cancer Res 60:150-5.

Bhowmick NA, Neilson EG, Moses HL (2004). Stromal fibroblasts in cancer initiation and progression. Nature 432:332-7

Bissell MJ, Radisky D (2001). Putting tumours in context. Nat Rev Cancer 1:46-54.

Braga VM (2002). Cell-cell adhesion and signalling. Curr Opin Cell Biol 14:546-56.

Brugghe J, Baak JP, Meijer GA, van Diest PJ, Brinkhuis M (1998). Rapid and reliable assessment of volume percentage of epithelium in borderline and invasive ovarian tumors. Anal Quant Cytol Histol 20:14-20. 
Castro CM, Yang Y, Zhang Z, Linnoila RI (2000). Attenuation of pulmonary neuroendocrine differentiation in mice lacking Clara cell secretory protein. Lab Invest 80:1533-40.

Chantrain CF, DeClerck YA, Groshen S, McNamara G (2003). Computerized quantification of tissue vascularization using high-resolution slide scanning of whole tumor sections. J Histochem Cytochem 51:151-158.

Cross SS (1997). Fractals in pathology. J Pathol 182:1-8.

Deering RE, Bigler SA, King J, Choongkittaworn M, Aramburu E, Brawer MK (1994). Morphometric quantitation of stroma in human benign prostatic hyperplasia. Urology 44:64-70.

Elie N, Plancoulaine B, Signolle JP, Herlin P (2003). A simple way of quantifying immunostained cell nuclei on the whole histologic section. Cytometry A 56:37-45.

Hicklin DJ, Witte L, Zhu Z, Liao F, Wu Y, Li Y, Bohlen P (2001). Monoclonal antibody strategies to block angiogenesis. Drug Discov Today 6:517-28.

Johansson AC, Visse E, Widegren B, Sjogren HO, Siesjo P (2001). Computerized image analysis as a tool to quantify infiltrating leukocytes: a comparison between high- and low-magnification images. J Histochem Cytochem 49:1073-9.

Kamate C, Baloul S, Grootenboer S, Pessis E, Chevrot A, Tulliez M, Marchiol C, Viguier M, Fradelizi D (2002). Inflammation and cancer, the mastocytoma P815 tumor model revisited: triggering of macrophage activation in vivo with pro-tumorigenic consequences. Int J Cancer 100:571-9.

Katsoulis M, Lekka J, Vlachonikolis I, Delides GS (1995). The prognostic value of morphometry in advanced epithelial ovarian cancers. Br J Cancer 72:958-63.

Kerbel R, Folkman J (2002). Clinical translation of angiogenesis inhibitors. Nat Rev Cancer 2:727-39.

Kim NT, Elie N, Plancoulaine B, Herlin P, Coster M (2003). An original approach for quantification of blood vessels on the whole tumour section. Anal Cell Pathol 25:63-75.

Konemann S, Schuck A, Malath J, Rupek T, Horn K, Baumann M, Vormoor J, Rube C, Willich N (2000). Cell heterogeneity and subpopulations in solid tumors characterized by simultaneous immunophenotyping and DNA content analysis. Cytometry 41:172-7.

Krout KE, Jenkins JM, Loewy AD (2002). High-resolution scanner for neuroanatomical analysis. J Neurosci Methods 113:37-40.

Liotta LA, Kohn EC (2001). The microenvironment of the tumour-host interface. Nature 411:375-9.

Matrisian LM, Cunha GR, Mohla S (2001). Epithelialstromal interactions and tumor progression: meeting summary and future directions. Cancer Res 61:3844-6.

Mueller MM, Fusenig NE (2004). Friends or foes - bipolar effects of the tumour stroma in cancer. Nat Rev Cancer 4:839-49.
Novak CL, Shafer SA (1992). Color vision. In: Shapiro SC. Encyclopedia of Artificial Intelligence. New York: Wiley-Interscience, 192-202.

Schipper NW, Baak JP, Smeulders AW (1991). Automated selection of the most epithelium-rich areas in gynecologic tumor sections. Anal Quant Cytol Histol 13:395-402.

Schmitt O, Eggers R (1999). Flat-bed scanning as a tool for quantitative neuroimaging. J Microsc 196(3):337-46.

Seiki M (2002). The cell surface: the stage for matrix metalloproteinase regulation of migration. Curr Opin Cell Biol 14:624-32.

Shacter E, Weitzman SA (2002). Chronic inflammation and cancer. Oncology (Huntingt) 16:217-26, 229.

Shapiro E, Becich MJ, Hartanto V, Lepor H (1992). The relative proportion of stromal and epithelial hyperplasia is related to the development of symptomatic benign prostate hyperplasia. J Urol 147:1293-7.

Signolle N, Francoise R, Elie N, Bosq J, Herlin P (2003). Chips and cheap: low resolution tissue array image analysis. Anal Cell Pathol 25:77.

Smolle J (1998). Fractal tumor stromal border in a nonequilibrium growth model. Anal Quant Cytol Histol 20:7-13

Sokoloff MH, Chung LW (1998). Targeting angiogenic pathways involving tumor-stromal interaction to treat advanced human prostate cancer. Cancer Metastasis Rev 17:307-15.

Song Z, Powell WC, Kasahara N, van Bokhoven A, Miller GJ, Roy-Burman P (2000). The effect of fibroblast growth factor 8 , isoform $\mathrm{b}$, on the biology of prostate carcinoma cells and their interaction with stromal cells. Cancer Res 60:6730-6.

Thuillier J, Plancoulaine B, Lezoray O, Herlin P, Bloyet D (2001). D Building of a microscopical image acquisition device dedicated to clinical oncology. Anal Cell Pathol 22:77.

Tsai W (1985). Moment-Preserving Thresholding: a New Approach. Computer Vision, Graphics, and Image Processing 29:377-93

Weiss L (2000). Metastasis of cancer: a conceptual history from antiquity to the 1990s. Cancer Metastasis Rev 19:193-383.

Yamada K, Kemler R (2002). Cell-to-cell contact and extracellular matrix. Curr Opin Cell Biol 14:527-30.

Yang Y, Shuaib A, Li Q (1998). Quantification of infarct size on focal cerebral ischemia model of rats using a simple and economical method. J Neurosci Methods 84:9-16.

Zhu B, Block NL, Lokeshwar BL (1999). Interaction between stromal cells and tumor cells induces chemoresistance and matrix metalloproteinase secretion. Ann N Y Acad Sci 878:642-6. 